\title{
Sub-clinical levels of attention deficit-hyperactivity disorder are associated with tobacco consumption in male but not in female smokers
}

\author{
Rebecca L. Douglas, Sean P. Barrett*, Neil T. Hanley and Robert O. Pihl
}

\begin{abstract}
An abudance of evidence has demonstrated an association between symptoms of attention deficit hyperactivity disorder (ADHD) and tobacco consumption. However, previous research has focused solely on populations meeting full diagnostic criteria for ADHD, despite evidence suggesting that symptoms below diagnostic threshold can be associated with impairment. Furthermore, the role of gender in the relationship between ADHD symptoms and tobacco consumption has not been determined. To examine the relationship between ADHD symptoms, tobacco use, and gender in a non-clinical population, symptoms of inattention, hyperactivity and impulsivity were assessed in 230 undergraduate students (22 male and 45 female smokers, and 66 male and 97 female nonsmokers). Overall, relative to nonsmokers, the smoking subjects reported significantly higher levels of inattention and hyperactivity. In male smokers, both inattentive and hyperactive/impulsive symptoms were positively associated with the number of cigarettes smoked daily. This relationship did not hold for female smokers, for whom no association was found between symptoms and nicotine consumption. Findings imply that even sub-clinical levels of inattention and hyperactivity/impulsivity are related to indices of tobacco use in males, and support previous research suggesting that significant gender differences may exist in tobacco smoking motives. Results also have potential implications for tobacco cessation programs, which may require more individual tailoring.l glutamic pyruvic transaminase is identified in an elderly subject.
\end{abstract}

\section{INTRODUCTION}

Attention deficit hyperactivity disorder (ADHD) is a pervasive psychiatric disorder, characterized by symptoms of inattention and/or hyperactivityimpulsivity. The Diagnostic and Statistical Manual of Mental Disorders (DSM-IV) currently recognizes three subtypes of ADHD: (i) a hyperactive-impulsive type, characterized by excessive motor activity and a

* To whom correspondence should be addressed: Sean P. Barrett, Department of Psychology, McGill University, 1205 Doctor Penfield, Montreal, Quebec Canada, H3A 1B1. Fax: (514) 3984896; Phone : (514) 398-6119; e-mail: barrett@ego.psych.mcgill.ca difficulty in delaying responses, (ii) an inattentive type, in which individuals display a failure to pay close attention to detail or to sustain attention, and (iii) a combined type that includes features of both hyperactivity-impulsivity and inattention (1). Although traditionally perceived as a form of childhood pathology, it is now widely recognized that ADHD often persists into adulthood (2-4). In order for an individual to receive a diagnosis of ADHD, six out of nine criteria for inattention and/or hyperactivity/impulsivity must be met (1). Individuals presenting with fewer than six criteria do not receive a diagnosis of ADHD, regardless of the degree of 
impairment (1). As no physiological significance has been attached to the cutoff criteria $(5,6)$, the diagnostic threshold for ADHD is potentially arbitrary. In fact, problematic levels of disruptive behavior associated with hyperactivity and impulsivity seem to occur even when the symptom count does not meet the diagnostic threshold $(7,6)$, indicating that the impairment associated with the disorder is not categorical. Although evidence suggests that significant dysfunction can be associated with sub-threshold levels of ADHD symptomatology $(6,7)$, little is known about how 'nonclinically significant' levels of hyperactivity and/or inattention relate to other forms of pathology. Because ADHD has been associated with a range of substance use problems, such as marijuana, cocaine, and other stimulant drug use disorders (8), as well as conduct problems, mood disorders, anxiety disorders, and learning disabilities $(2,3)$, it remains possible that the presence of even sub-clinical symptoms may increase an individual's risk for certain adverse outcomes, including substance use and other psychiatric disorders.

An abundance of evidence suggests a link between the presence of ADHD symptoms and tobacco usage. ADHD in childhood has been associated with significantly younger age of first tobacco use (9) and younger age of commencement of daily tobacco consumption (10). In addition, a greater proportion of adults with ADHD display tobacco dependence relative to controls $(11,10)$. The relationship between tobacco consumption and symptoms of inattention and hyperactivity/impulsivity may be partially or completely explained by attempts to self-medicate symptoms of ADHD. Nicotine has been demonstrated to ameliorate both inattention and hyperactive/impulsive behavior $(12,13)$. Nicotine has also been demonstrated to induce cognitive improvements, mostly in attentional areas, in normal nonsmoking adults as well as those with impairments (13). Even low doses of nicotine have been shown to significantly reduce errors of omission on attentional tasks, without increasing errors of commission, thus indicating that the effects are not simply due to increased responding (13). These effects do not appear to diminish with chronic administration (13). Significant improvements in memory performance, specifically working memory, have also been shown with nicotine administration over long periods of time (13).

The various effects of nicotine on attention and behaviours related to hyperactivity/impulsivity may be linked to nicotine's effect on dopamine transmission. Nicotine indirectly facilitates dopamine transmission, through stimulation of the nicotinic acetylcholine receptors located on dopamine containing neurons (14,
15). Studies of smokers have shown that nicotine seems to inhibit monoamine oxidase-B (MAO-B), thus increasing the availability of dopamine in the brain by preventing breakdown (11). Nicotine is also believed to exert its effect on working memory and on regulation of impulsive behaviors by interacting with the dopaminergic systems and receptors in the brain (11, 13).

Because ADHD symptoms are thought to result from dopamine dysfunction particularly in frontal and striatial regions (2), it seems possible that individuals who display greater inattention or hyperactivity/impulsivity may use tobacco in an attempt to address these symptoms. Previous research has in fact suggested that tobacco may be used for selfmedication in clinical ADHD populations (10), and as sub-threshold symptoms of inattention and hyperactivity/impulsivity also result in impairment $(6,7)$, there may be motivation for individuals, even in non-clinical populations, to self-medicate.

There is some evidence to suggest that gender differences may exist in the relationship between smoking and ADHD symptoms. It has been suggested that females may be less sensitive to nicotine's effects (17); the lower efficacy of nicotine replacement therapy found among females in smoking cessation programs suggests a decreased sensitivity in females to the negative reinforcement effects of nicotine (17). Males also appear to be more likely to self-administer nicotine (17). The findings of gender specific nicotine effects raise the possibility of differences in likelihood to selfmedicate ADHD symptoms with tobacco.

To date, research that links tobacco smoking with symptoms of ADHD has focused on participants whose symptoms meet the full diagnostic criteria for ADHD. Although such symptoms fall on a continuum (5), with concomitant dysfunction even at levels below the diagnostic threshold $(6,7)$, the degree to which subclinical levels of either inattention or hyperactivity/impulsivity may be related to tobacco smoking behavior is not known. Furthermore, although there appears to be significant gender differences in the manifestation of hyperactive/impulsive and inattentive symptoms (16), previous studies examining the link between ADHD and tobacco smoking have either used male participants exclusively (9) or have failed to analyze male and female participants separately (10). Thus, the relationship between inattention and/or hyperactivity/impulsivity symptoms and tobacco consumption remains unknown in females.

The purpose of this study was to examine the relationship between inattention, hyperactivity/ impulsivity, and tobacco consumption in a non-clinical population, and to determine how this relationship may 
differ with respect to gender.

\section{METHOD}

Two hundred forty-four undergraduate university students volunteered to participate in the study. Potential participants were excluded on the basis of past or present ADHD or other psychiatric diagnoses, however the use of illicit drugs was not an exclusion criterion. In total, 14 participants were eliminated for not meeting the study's inclusion criteria. Volunteers were approached at various locations at the McGill University campus and were asked to complete anonymous questionnaires. The questionnaire consisted of an eighteen-item checklist for ADHD symptoms, as defined by the DSM-IV (1), consisting of nine items each for inattention and hyperactivity/impulsivity. Because the DSM suggests an assessment of childhood symptoms in adults with ADHD (1) a nineteenth item asked participants to rate their level of childhood hyperactivity. Similar self-report check-lists are frequently used in clinical practice for the assessment of ADHD in adults (e.g. 18, 19) and this method of ascertaining current and childhood symptoms of ADHD is widely considered to be reliable and valid in both clinical $(18,19)$ and non-clinical populations (20). Participants were asked to indicate the degree to which each item applied to them using visual analogue scales. Based on their self-reported endorsement of symptoms, each participant was assigned a total composite ADHD score, as well as inattention and hyperactivity /impulsivity sub-scores, based on DSM-IV ADHD diagnostic criteria (1). While possible scores ranged from zero to ten on all item scales, with higher scores reflecting greater symptomatology, scores were converted to z-scores before calculating composite scores in order to avoid excessive influence of items with a limited range of responses. Therefore, means reported for overall symptoms and the two sub-scores appear as z-scores. In addition to completing the ADHD self-report scales, participants also reported on their lifetime use of tobacco. Participants were classified as current smoker, never smoker, or former smoker. In order to control for the potential confounding factor of smoking cessation, 15 former smokers were eliminated from the analysis. In addition to reporting their smoking status, smokers also reported the average number of cigarettes typically consumed daily. All participants were blinded to the specific hypotheses of the study, but the investigators were not.

Pearson bivariate correlations were performed in order to determine the relationship between measures of tobacco consumption and ADHD symptomatology, while independent t-tests were performed to examine potential effects of gender and smoking status.

\section{RESULTS}

Symptoms of inattention and hyperactivity or impulsivity were assessed in a non-clinical sample of 67 smokers (67\% female) and 148 nonsmokers (60\% female) with a mean age of 21.57 years $(S D=2.55$, range 18 to 30 ). Males and females did not significantly differ in their current smoking status $(26.8 \%$ vs. $33.8 \%$ classified as current smokers; $2(1, \mathrm{~N}=215)=1.15$, $\mathrm{p}=.760$, NS), nor did male and female smokers differ in their average number of cigarettes smoked per day (males $\mathrm{M}=5.86, \mathrm{SD}=8.27$; females $\mathrm{M}=4.46, \mathrm{SD}=$ $7.17 ; \mathrm{t}(65)=.65, \mathrm{p}=.479, \mathrm{NS})$.

The analyses revealed significant differences between smokers and nonsmokers on the measures of overall symptoms as well as inattention and hyperactivity/impulsivity separately. Current smokers reported greater overall ADHD symptoms $(\mathrm{M}=.09, \mathrm{SD}$ $=.44)$ than did nonsmokers $(\mathrm{M}=-.07, \mathrm{SD}=.37 ; \mathrm{t}(211)$ $=-2.74, \mathrm{p}=.007)$. Smokers rated themselves as experiencing higher symptoms of inattention $(\mathrm{M}=.11$, $\mathrm{SD}=.51)$ than nonsmokers $(\mathrm{M}=-.08, \mathrm{SD}=.49 ; \mathrm{t}(212)$ $=-2.29, \mathrm{p}=.023)$ as well as having higher hyperactivity/impulsivity (smokers $\mathrm{M}=.08, \mathrm{SD}=.49$; nonsmokers $\mathrm{M}=-.07, \mathrm{SD}=.45 ; \mathrm{t}(140)=-2.17, \mathrm{p}=$ $.031)$.

Within male and female smokers combined, only symptoms of hyperactivity/impulsivity were related to level of tobacco consumption $(\mathrm{r}(67)=.254, \mathrm{p}=.038)$. However, when analyzed by gender, it was determined that the relationship found between smoking and level of ADHD symptoms differed between males and females. While daily consumption was strongly associated with overall symptoms $(\mathrm{r}(22)=.595, \mathrm{p}=$ $.003)$, inattention $(\mathrm{r}(22)=.483, \mathrm{p}=.023)$ and hyperactivity/impulsivity $(\mathrm{r}(22)=.554, \mathrm{p}=.007)$ in males, there were no significant relationships between smoking and ADHD symptoms in females (ps > .100, NS).

Finally, the relationship between the degree of childhood hyperactivity and current cigarette consumption was assessed. With male smokers, there was a modest but non-significant association between childhood hyperactivity and current daily consumption $(\mathrm{r}(22)=.391, \mathrm{p}=.07)$, whereas this association was not observed in female smokers $(\mathrm{r}(45)=.007, \mathrm{p}=.961, \mathrm{NS})$.

\section{DISCUSSION}

Based on previous findings of increased tobacco use in clinical ADHD populations (e.g. 9) and the recognition that $\mathrm{ADHD}$ symptoms fall on a continuum $(5,6)$ with impairment occurring at sub-diagnostic levels $(6,7)$, it was hypothesized that tobacco use would be positively associated with inattention and 
hyperactivity/impulsivity in a non-clinical sample.

Indeed, significant differences in levels of both inattention and hyperactivity/impulsivity were found between smokers and nonsmokers, with smokers displaying much greater levels of each sub-score. Amongst smokers, daily consumption was positively associated with both inattention and hyperactivity/impulsivity in males. Interestingly however, no significant relationships were found among female participants.

In an attempt to discern the direction of the relationship between ADHD symptoms and tobacco consumption, temporal order was addressed. Although not significant, an association was identified between the reported level of childhood hyperactivity and current tobacco consumption in male smokers. No parallel association was found with female smokers.

The relationship between smoking and symptoms of inattention and hyperactivity/impulsivity found in males may be due to an attempt to self-medicate symptoms of ADHD. Males with more ADHD symptoms may have higher nicotine consumption due to nicotine's positive effects on attention and symptoms of hyperactivity/impulsivity via its indirect effects on the dopaminergic system.

The dramatic gender differences found in the relationships between tobacco consumption and ADHD symptomatology add a further layer of complexity. One possibility for these observed differences is that males alone self-medicate with tobacco. This possibility is supported by the findings that suggest gender differences in the motivation for tobacco consumption and maintenance (17). The current findings are consistent with the evidence that females may be less sensitive to nicotine's effects (16). Given the gender specific effects of nicotine and the proposed gender differences in smoking motives, women may be less likely to self-medicate with tobacco for ADHD symptomatology, as it is the nicotine component in tobacco that is suggested to influence symptoms of ADHD.

There are several implications for the findings of this study. First, the finding that smoking is associated with inattention and hyperactivity/impulsivity even in a nonclinical population supports previous research that has found functional impairment in those below the diagnostic threshold of ADHD symptomatology $(6,7)$. Second, there is support for previous research reporting significant gender differences in tobacco smoking motives. Both these findings have implications for the prevention and treatment of tobacco consumption for those with ADHD symptoms. Individual differences, for example in levels of inattention and hyperactivity/impulsivity, may be factors that need to be considered in designing programs for both prevention and treatment of tobacco consumption. If smoking functions to self-medicate the ADHD symptoms of some individuals, then employing other methods to medicate or manage these symptoms may facilitate smoking cessation. Furthermore, cessation programs may need to be tailored by gender in order to target the appropriate smoking motives.

Future research is needed to further examine this phenomenon of self-medication with tobacco in those with clinical, as well as non-clinical, ADHD symptomatology, as well as to examine possible gender differences in tobacco usage and the effects on symptoms within this population.

\section{REFERENCES}

1. American Psychiatric Association. Diagnostic and StatisticalManual of Mental Disorders, 4th ed.. Washington, DC: American Psychiatric Association, 1994.

2. Biederman J. Attention-Deficit/Hyperactivity Disorder: A Life Span Perspective. Journal of Clinical Psychiatry, 59: 4-16; 1998.

3. Troller JN. Attention Deficit Hyperactivity Disorder in Adults: Conceptual and Clinical Issues. Medical Journal of Australia, 171: 421-425; 1999.

4. Wender PH. Attention-Deficit Hyperactivity Disorder in Adults. The Psychiatric Clinics of North America, 21: 761-774; 1998.

5. Levy F, Hay D, McStephen M, Wood C, Waldman I. AttentionDeficit Hyperactivity Disorder: A Category or a Continuum? Genetic Analysis of a Large-scale Twin Study. Journal of American Academy of Child and Adolescent Psychiatry, 36 :737-744; 1997.

6. Neuman RJ, Todd RD, Heath AC, et al. Evaluation of ADHD Typology in Three Contrasting Samples: A Latent Class Approach. Journal of the American Academy of Child and Adolescent Psychiatry, 38, 25-33; 1999.

7. Hudziak J, Heath A, Madden P et al. The Latent Class and Factor Analysis of DSM-IV ADHD: A Twin Study of Female Adolescents. Journal of the American Academy of Child and Adolescent Psychiatry, 37: 848-857; 1998.

8. Biederman J, Wilens T, Mick E, Milberger S, Spencer TJ, Faraone SV. Psychoactive Substance Use Disorders in Adults with Attention Deficit Hyperactivity Disorder (ADHD): Effects of ADHD and Psychiatric Comorbidity. American Journal of Psychiatry, 152: 1652-1658; 1995.

9. Milberger S, Biederman J, Faraone SV, Chen L, Jones J. ADHD is Associated With Early Initiation of Cigarette Smoking in Children and Adolescents. Journal of the American Academy of Child and Adolescent Psychiatry, 36: 37-44; 1997.

10. Lambert NM. \& Hartsough CS. Prospective Study of Tobacco Smoking and Substance Disorders Among Samples of ADHD and Non-ADHD Participants. Journal of Learning Disabilities, 31: 533-544; 1998.

11. Hornig M. Addressing Comorbidity in Adults with AttentionDeficit/Hyperactivity Disorder. Journal of Clinical Psychiatry, 59: 69-75; 1998.

12. Koelega HS. Stimulant Drugs and Vigilance Performance: A Review. Psychopharmacology, 111:1-16; 1993.

13. Levin ED \& Rezvani AH. Development of Nicotinic Drug Therapy for Cognitive Disorders. European Journal of Pharmacology, 393: 141-146; 2000.

14. Wonnacott S, Kaiser S, Mogg A, Soliakor 1, Jones IW. 
Presynaptic Nicotinic Receptors Modulating Dopamine Release in the Rat Striatum. European Journal of Pharmacology, 393: 51-58; 2002.

15. Yu H, Matsubayashi H, Amano T, Cai J, Sasa M. Activation by Nicotine of Striatal Neurons Receiving Excitatory Input from the Substantia Nigra Via Dopamine Release. Brain Research, 872:223-226; 2000 .

16. Lahey BB, Applegate B, McBurnett K et al. DSM-IV Field Trials for Attention Deficit Hyperactivity disorder in Children and Adolescents. American Journal of Psychiatry, 151: 16731685; 1994.

17. Perkins KA, Donny E, Caggiula AR. Sex Differences in Nicotine Effects and Self-Administration: A Review of Human and Animal
Evidence. Nicotine \& Tobacco Research, 1: 301-315; 1999.

18. O'Donnell JP, McCann KK, Pluth S. Assessing Adult ADHD Using a Self-Report Symptom Checklist. Psychological Reports. Vol 88: 871-881; 2001.

19. Murphy P, Schachar R. Use of Self-Ratings in the Assessment of Symptoms of Attention Deficit Hyperactivity Disorder in Adults. American Journal of Psychiatry. Vol 157: 1156-1159; 2000 .

20. Mehringer, AM, Downey KK, Schuh LM, Pomerleau CS, Snedecor SM, Schubiner H. The Assessment of Hyperactivity and Attention: Development and Preliminary Validation of a Brief Self-Assessment of Adult ADHD. Journal of Attention Disorders. Vol 5: 223-231; 2002.

Rebecca Lynn Douglas is currently a first-year PhD student in clinical psychology at Dalhousie University. She received her BA in psychology from McGill University. Her research interests include psychopathy, the etiology of violent behaviour, and impulse control disorders. Sean Patrick Barrett is currently a fourth-year student in clinical psychology at McGill University. He holds a BA in psychology from Saint Francis Xavier University. His research interests include the neuropsychopharmacology of addictive drugs and psychiatric comorbidy. Neil Hanley is currently completing a PhD in clinical psychology at McGill University. He holds a BA from Simon Fraser University. His broad research interests include perceptions of control and the application of learning theory to anxiety and mood disorders. He will begin an internship at the Royal Victoria Hospital in the fall. 